\title{
Development of a fish leaping framework for low-head barriers
}

\author{
Parham A. Mirzaei*
}

Architecture and Built Environment Department, The University of Nottingham, Nottingham NG7 2RD, UK

\section{Abstract}

Leaping is an indispensable part of the upstream spawning migration of a fish species.

The natural barriers replaced with artificial dams and obstacles can obstruct the leaping process and destruct the life cycle of fish species, causing their extinction in extreme scenarios. To help design and improve the artificial barriers, many studies have been conducted to model the leaping success of fish species. However, generic results were scarcely obtained to be extended for a wide range of barriers. The main reasons can be identified as the lack of thorough understanding of the interaction between fish locomotion and water flow regime upstream of the investigated barriers. Hence, the aim of this study is to propose a leaping framework compatible with a diverse range of fish species and barriers. This framework includes a detailed hydraulic sub-model as well as locomotion model capable of tracing fish in both water and air environments. The functionality of the proposed framework is further discussed using a selected case study.

Keywords: Computational fluid dynamics, Fish jumping, Low-head dam, Spillway, Upstream spawning migration

\section{Introduction}

Environmental preservation is a challenging issue of the current century to mitigate the past, current, and future interference of human footprints. A recent example of the ongoing damage to the environment is fish species extinction due to the construction of geographical and physical barriers in rivers and waterways (Schlosser and Angermeier, 1995). Human fragmentation has resulted in a wide range of ecological problems such as local species extinction (Wilcox, 1980;

\footnotetext{
Correspondence: Parham A. Mirzaei, Tel.: $0115 \quad 95 \quad$ 14129, Fax: $\quad+44(0) \quad 115 \quad 951 \quad 3159$, $\quad$ Email: parham.mirzaei_ahranjani@nottingham.ac.uk
} 
Wilcox and Murphy, 1985). For example, fragmentation has been recognized as a cause of local extinction of small fishes in Australia (Gehrke et al., 2002).

Upstream spawning migration is a part of the life cycle of many fish species, e.g. pacific salmon, smelt, shad, striped bass, and sturgeon (McDowall, 1990). These fish are born in fresh waters, migrate downstream toward sea where they become mature in a period of a few years. Then, they return back to the same stream through a long upstream migration, and spawn in the fresh waters. A part of this upstream migration is jumping through the natural barriers. However, the artificial barriers such as road-crossings, rocks, weirs, and low head dams are common impassable obstacles, cutting the ecological connectivity of fish spawning migration.

Removal of the mentioned artificial structures is not always a practical and economical option. Therefore, as potential solutions, fish ladders, fish ways and passageways have been widely designed and constructed to help maintain the ecological life cycle of fish. Many design guidelines and instructions have been developed and implemented (Marmulla, 2001). Despite the positive functionality of the fish passageways, their effectiveness is sometimes questioned as the economical and engineering considerations are more dominant compared to the jumping ability and performance of fish species (McLaughlin et al. 2013; Noonan et al. 2012).

Various studies have been conducted to help better the understanding of the jumping ability and performance of different fish species, where the influential parameters are recognized as water flow rate, pool depth, fall height, fish body length, etc. As an early mathematical model, Reiser and Peacock (1985) calculated the maximum attainable height by a fish using the initial burst speed. Powers and Orsborn (1985) defined a more precise model by including further parameters such as maximum burst, fish length, fish frontal area, and estimated drag force. The main limitation of such model can be identified as their simplified hydraulics model.

In a more advanced study, Lauritzen et al. (2005) examined the jumping kinematic of wild sockeye salmon in natural waterfalls. They have observed the kinematic of fish jumping and developed a simple mathematical ballistic model based on the trajectory of fish in the air. They concluded that the height of waterfall and depth of pool below it are important factors in the 
jumping performance. The influence of environmental factors such as brown bear presence on the jumping success was further considered in this study. In another experimental study conducted in a water tank by Ficke et al. (2011), speed, performance and kinematics of Brassy Minnow fish were investigated for better design of fish ways. Beside the role of fish length, waterfall height, and pool depth, they have emphasised the role of water temperature in endurance jumping of Brassy Minnow. Moreover, the experiment indicated the significance of water velocity on the swimming endurance of studied species. Different regression equations of the probability of jumping success were developed based on the mentioned influential parameters. Furthermore, Salmon jumping was studied through an observational experiment conducted in an adjustable water tank (Lauritzen et al., 2010). The flow speed, pool depth, fall height and fall angels were changed to investigate the jumping success rate. In another laboratory experiment by Kondratieff and Myrick (2006), jumping performance of Brook trout was evaluated. Again, a regression model was developed based on the recognized influential parameters, including pool depth, waterfall height, fish length, trial duration, and fish condition. The impact of fish condition on leaping was assessed based on the fish level of damage in fins, jaw, eyes, and operculum condition.

Despite the various studies on understanding the relation between fish jumping success and environmental/physiological parameters, contradictory results were occasionally reported in the development of jumping models (Myrick et al., 2004). This implies that most of these models are restricted to the laboratory and simplified conditions, and barely can be generalized to a wider range of barriers with different physical characteristics. This weakness is inherently due to the simplified details of the utilized hydraulic models on the jumping ability of fish species. While the developed models mainly recognize the importance of barriers and fish characteristics on the jumping success, they barely represent their interconnections with water flow regime. In other words, the poorly modelled water flow regime, affected and formed by barrier geometries such as pool depth and water fall height, significantly impacts the kinematic of a fish species.

The fish kinematic is the ability of a fish species to benefit from water flow characteristics to minimize the locomotion cost and maximize the success probability of the jumping. Therefore, 
development of hydraulic models can provide more details about the interaction between a fish and its surrounding environment compared to the traditional regression and observational models. The advantage of such models can be addressed as their capability in resolving the turbulence level and circulation strength of the flow regime. Turbulence is identified as a significant factor in attraction or repelling a fish as it can dominantly decrease or increase the locomotion cost (Enders et al., 2003; Pavlov et al., 1982, 2000; Webb, 1998). The mentioned parameters are mainly neglected in the traditional models, resulting in limited conclusions extracted from these studies. In general, fish kinematic depends on the characteristics of its species in generating locomotion forces (i.e. drag, lift, thrust and buoyancy).

Species characteristics of a specific fish itself contain the physiological and behavioural parameters. While physiological parameters of a species (e.g. weight, length, shape) are independent of the flow regime, behavioural parameters are directly impacted by constraints of the flow regime, again justifying the implementation of a detailed flow model. Behavioural parameters include the maximum swimming speed (Reiser et al., 2006), visual ability (Sweka et al., 2001), temperature endurance (Holthe et al. 2005), environmental fear (Carpenter et al., 2009), and learning ability (Odling-Smee and Braithwaite, 2003). It is widely studied that fish use their sensory and locomotion systems to navigate efficiently within the water with changing their swimming kinematics according to the flow regime (Liao, 2007). Liao et al. (2003) showed how fish surf in water and use underwater vortices to minimize their swimming energy. Takagi et al. (2013) showed how Pacific Bluefin Tuna can reduce its locomotion cost through a glide and upward swimming rather than a continuous horizontal one. As another example, reported by Lauritzen et al. (2005), straightening bodies, closing mouth, stretching the fins, and continuous beating of the tail can be respectively justified as minimizing drag and maximizing thrust forces.

Hence, the aim of this study is to develop a framework, performing as a roadmap to develop simulation models of the jumping mechanism of various fish species over a variety of barriers. It should be noted again that the goal of this research is to explore the functionality of the proposed framework rather than generating reliable results for a specific fish species, which requires a thorough calibration with that of realistic behavioural and physiological parameters. The 
proposed framework can be later used to evaluate the jumping favourability of any low-head barrier and to calculate the total energy budget of a fish species through several jumps required for its spawning migration. The proposed framework consists of flow details as well as physiological and behavioural characteristics of a fish species. Furthermore, it contains a submodel to trace the fish trajectory in the air when it departs the water surface. The functionality of the proposed framework and its sub-models are shown with a selected case study.

\section{Jumping mechanism}

The proposed framework, encompassing the mentioned flow and fish parameters of the jumping, is exhibited in Fig. 1. The framework describes the dynamic interaction between a fish and its environment. After being positioned at the initial point of a jump, a fish tries to benefit from its physiological abilities (i.e. weight, volume, and hydrodynamic) against the water flow regime of a pool to generate an optimum thrust force and swim angle, heading toward the water surface. During the burst process, a fish continuously adjusts its thrust force and swim angle to achieve a successful jump. The latter behavioural ability is unique for each fish species and corresponds to its eyesight, response time, and learning rate. In general, fish optimal solution on a specific situation can result in a failure in jump due to the miscalculation in the jumping process related to its species and also to micro flow complexity associated with the low-head barrier. Therefore, the proposed framework attempts to simulate the jumping process from the fish species point of view. state while it is decoupled from the fish water kinematic model. This implies that CFD model only provides the flow characteristic to be inserted as inputs into the fish water kinematic model. Then, the flow field simulated by the hydraulic CFD model, in addition to physiological 
parameters of the fish species, are transferred to the second sub-model, the fish water kinematic

137

138

139

140

141

142

143

144

model to calculate the hydrodynamic forces; the fish trajectory is simulated as a particle trajectory. The hydrodynamic forces in addition with the behavioural abilities again serve as inputs to the third sub-model, the swimming optimization model, which calculates the minimal energy-consumption path from fish point of view. Thus, at this point, the fish is able to generate a thrust force and swim with a certain angle to reach the water surface. This dictates that the fish reaches a new location between its decision and response time when the fish again analyses the environment to either maintain or adjust the former swimming pattern. The fish continues the latter process of decision making based on its expected minimal energy consumption until reaching the water surface, where it enters the air with a particular velocity and angle, implying that the fish departs the water and enters the air. This stage is further modelled with a supplementary fish air trajectory model, the forth sub-model, to calculate the failure or success of the fish in reaching the upstream reservoir. At this level, fish slightly can enhance its jump quality with beating of its tail.

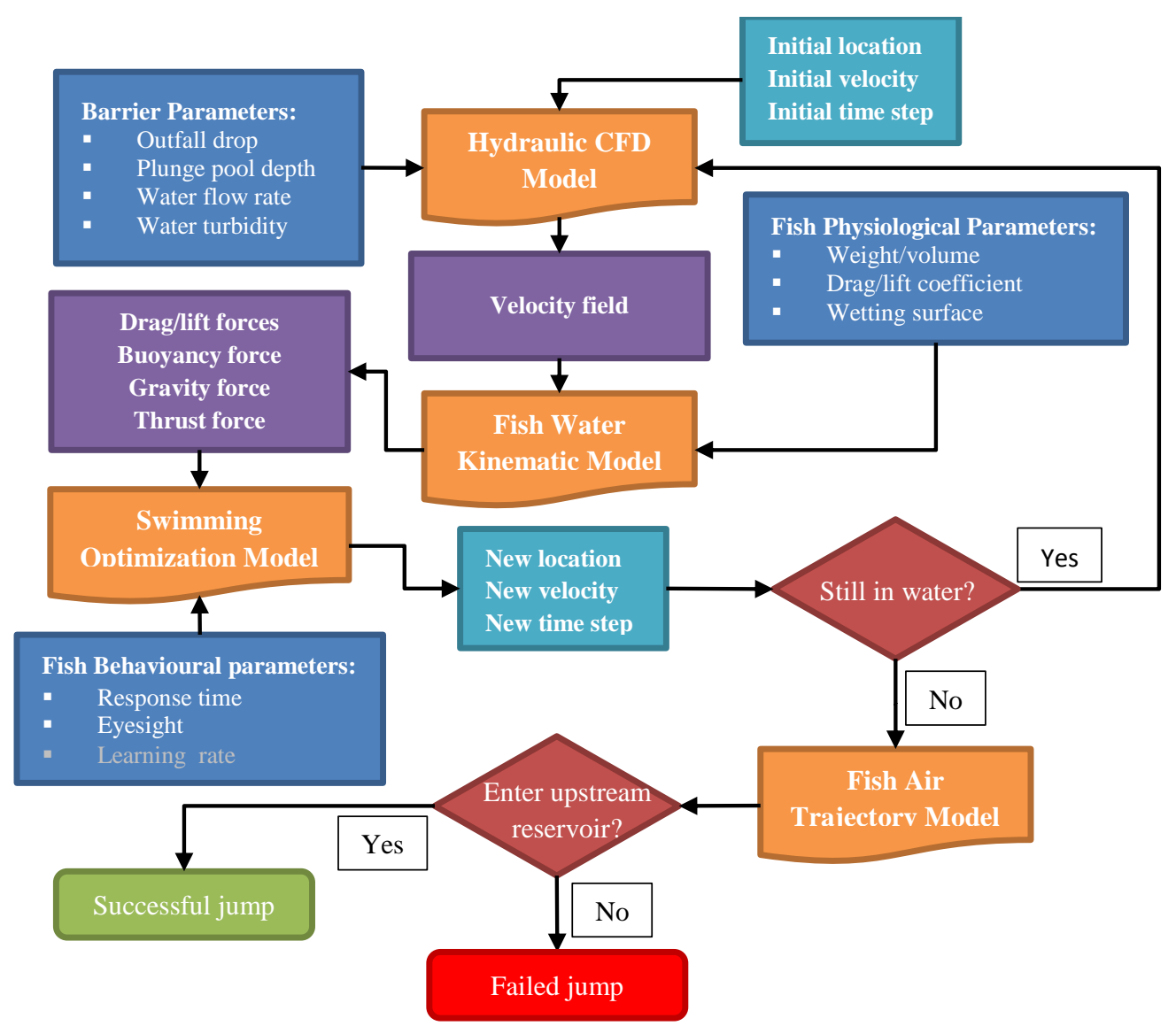

Figure 1 The proposed jumping framework of a fish species consists of four sub-models: (1) hydraulic CFD, (2) fish water kinematic, (3) swimming optimization, and (4) fish air trajectory models 


\section{Structure of the sub-models}

\subsection{Hydraulic CFD model}

The hydraulic model simulates the water flow regime to later be utilized in calculating the propulsion forces of a fish species. As stated before, any alteration in the low-head barrier design as well as the upstream water velocity impacts the flow regime that eventually affect the decision making process of the fish species. To simulate the flow regime, CFD as a widely accepted and powerful method is utilized to model the waterfall downstream of various types of barriers. The time-dependent flow fluctuation can be neglected and the water flow can thus be simulated under the steady state condition. Therefore, the water-air interface is determined by solving the transport equation of volume fraction given as follows:

$\frac{\partial}{\partial x_{j}}\left(C_{i} u_{j}\right)=0$

where $C_{i}$ is the fraction of each fluid and $u_{j}$ is the velocity in $j^{\text {th }}$ direction. The flow of viscous fluid is governed by Navier-Stokes (NS). The Reynolds-averaged approach is used to decompose the Navier-Stokes (NS) equations into mean and fluctuating terms (Mirzaei and rad, 2013). Therefore, in Cartesian coordinates, the governing equations can be written as follows:

$\frac{\partial}{\partial x_{i}}\left(\rho u_{i}\right)=0$

$\frac{\partial}{\partial x_{j}}\left(\rho u_{j} u_{i}\right)=-\frac{\partial p}{\partial x_{i}}+\frac{\partial}{\partial x_{j}}\left[+\mu\left(\frac{\partial u_{i}}{\partial x_{j}}+\frac{\partial u_{j}}{\partial x_{i}}\right)-\rho \overline{u_{\imath}^{\prime} u_{j}^{\prime}}\right]+\rho f_{i}$

where the source term, $f_{i}$, represents the surface tension and gravity force applied on the control volume in the $i^{\text {th }}$ direction. In terms of surface tension, the continuum surface force (CSF) model can be used to depict the interaction between water and air:

$F_{C S F}=\sigma_{i j} \frac{\rho k_{i} \nabla C_{i}}{\frac{1}{2}\left(\rho_{i}+\rho_{j}\right)}$

where $C_{i}$ is the volume fraction, $k_{i}$ is the curvature of free surface, and $\rho$ is the volumeaverage density based on the volume fraction. $\sigma$ is the surface tension coefficient. For the Reynolds stress components, $\overline{u_{\imath}^{\prime} u_{\jmath}^{\prime}}$, the Boussinesq hypothesis was employed. Moreover, turbulent kinetic energy and its dissipation rate were introduced to close the RANS equation (Mirzaei and Haghighat, 2013). ANSYS Fluent software was utilized to solve the NS equations. 


\subsection{Fish water kinematic model}

Although fish trajectory model utilizes the characteristics of the flow as its input, it is

180

181

182

183

184

185

186

187

188

189

190

191

192

193

194

200

201

inherently decoupled from the CFD model, meaning that the fish species is neglected in the CFD model as it has relatively small impact on the flow regime; inversely, it is highly impacted by water flow regime. Based on Weihs (1973), fish swimming is governed by thrust, drag, lift, buoyancy, and gravity (Fig. 2) as follows:

$\vec{T}=\vec{D}+\vec{L}+\vec{B}+\vec{W}-m \frac{d \vec{U}}{d t}$

where $\vec{T}$ is the thrust force. $\vec{D}$ and $\vec{L}$ are respectively the drag and lift forces. $\vec{B}$ stands for the buoyancy force and $\vec{W}$ denotes the weight of the fish species. $\vec{U}$ represents the fish absolute velocity, which is the sum of fish swimming velocity and water velocity. Thus, the last term in the right hand side presents the momentum of the fish. The drag force can be obtained with the following equation:

$\vec{D}=-\frac{1}{2} \rho S C_{D}\left|\vec{U}_{f}-\vec{U}_{w}\right|^{2} \frac{\vec{U}_{f}}{\left|\vec{U}_{f}\right|}$

where $\rho$ is the water density and $S$ is the wetting surface area. The $\vec{U}_{f}$ and $\vec{U}_{w}$ respectively represent the fish swimming velocity and water velocity. $C_{D}$ is the drag coefficient and is assumed to be independent from the swimming speed. Similarly, the lift force can be defined as below:

$\vec{L}=\frac{1}{2} \rho S C_{L}\left|\vec{U}_{f}-\vec{U}_{w}\right|^{2} \frac{\vec{U}_{f}}{\left|\vec{U}_{f}\right|}$

5 where $C_{L}$ is the lift coefficient and the rotation matrix indicates the direction of the force, which is normal to the fish swimming velocity. Drag and lift coefficients are chosen to be a function of attack angle and total body length, which are extracted from a look-up table created from a study by Takagi et al. (2013). Additionally, the buoyancy and gravity forces can be taken into consideration with the following equations:

$\vec{B}=-\rho V \vec{g}$

$\vec{W}=m \vec{g}$ 
203

204

205 species, respectively. Finally, the energy consumption caused by thrust force can be derived by integration of the thrust force along the swimming path:

$E=\int_{0}^{t} \vec{T} \cdot \vec{U}_{f} d t$

It should be noted that this energy can only represent the kinetic cost of transport, and the actual metabolic energy consumption is neglected in this study.

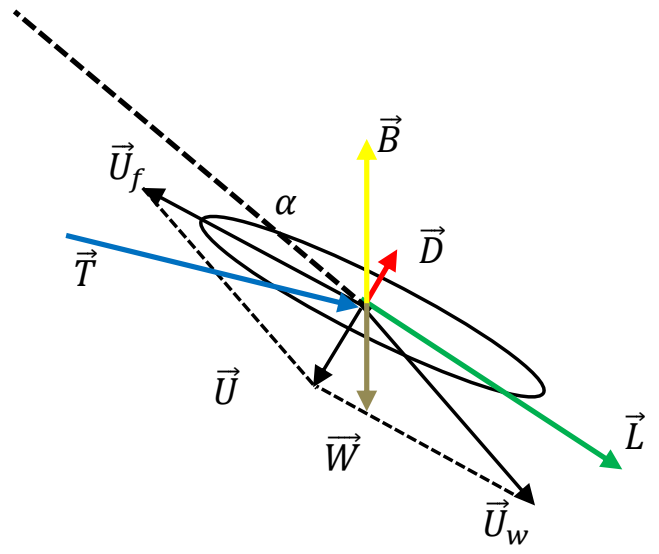

Figure 2 Kinematic of fish locomotion in water

\subsection{Swimming optimisation model}

After detecting the barrier, the fish has to respond quickly to avoid the obstacle, so it starts to change its swimming direction and speed. As it is stated in literature, fish follow the optimal solution for its jump in accordance with the information obtained from the surrounding environment. The path alteration of fish, however, cannot occur instantly, meaning that the response lag is inevitable. Therefore, the targeted leaping point is adjusted at each time step in response of the variation in water velocity and fish location. This implies that the fish's actual moving path will deviate from its preliminary decided path and is thus created in various time steps.

Hence, the purpose of swimming optimisation is to simulate fish decision and calculate the optimum thrust force, and minimising kinetic energy required for fish to leap over the barrier in each time step associated with the decision made by fish. It is noteworthy to mention that the decided path by fish occurs in response to its understanding from the flow characteristics and 
222

223

position from the spillway while the learning process is neglected in this study. The swimming optimisation is based on the assumption that fish sense the water flow at a certain distance and will move toward that point as shown in Fig.3. The decision process is defined based on the various angles and velocities that the fish species can select to reach the water surface. However, to minimize the calculation cost of the simulation, only three nodes were generated in each time step where the angle between thrust force and fish swimming velocity was restricted to be less than 30 degrees (Pearson et al. 2005). After a distance swum during its response lag (time step), fish again re-decide to maintain the current path or detour where three new nodes are generated as the possible direction of fish. Therefore, in each time step, expected leaping point and swimming angle and velocity will be adjusted by the fish species.
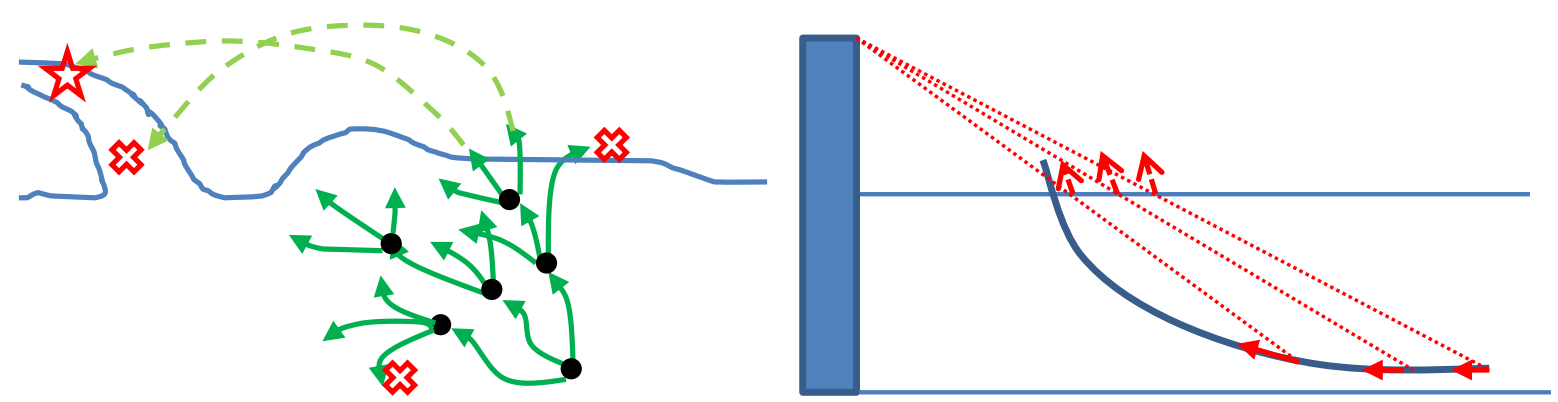

Figure 3 (right) local decided path to impact the water surface (left) simplified 3-nodes path optimization for the fish species

The water flow is assumed to be uniform at each time step to ensure a local optimal value for the thrust force. In this study, MATLAB optimisation toolbox was linked with the hydraulic CFD model used to solve the equations 5-9. In the optimisation algorithm, both the linear and non-linear constraints were applied to the calculation.

\section{4. $\quad$ Fish air trajectory model}

After departing the water surface, the motion of fish can be described as projectile motion in the air. The equation of this curved motion can be presented as follows (Powers and Orsborn, 1985):

$H_{L}=\tan \left(\theta_{L}\right) X_{L}-\frac{g X_{L}^{2}}{2\left(V_{F} \cos \theta_{L}\right)^{2}}$ 
where $H_{L}$ and $X_{L}$ are respectively the vertical and horizontal distances. $V_{F}$ denotes the

fish speed, and $\theta_{L}$ is the angle of leap (take-off) from the plunge pool. It should be noted that the small thrust force generated by the rapid tail flapping of fish is neglected in this study.

\section{Case Study}

To further investigate the jumping mechanism over a spillway as an artificial barrier, a standard spillway designed by the U.S. Army Corps of Engineers (1990) was selected as the case study (Fig. 4a). The shape of the overflow spillways and the flow pattern is parameterised by three influential parameters, including the design head $\left(\mathrm{H}_{\mathrm{d}}\right)$, water flow rate $(\mathrm{Q})$, and the slope (k). In this study, the spillway characteristics were respectively selected to be $\mathrm{R}_{1}=0.5 \mathrm{H}_{\mathrm{d}}, \mathrm{R}_{2}=$ $0.2 \mathrm{H}_{\mathrm{d}}$, and $\mathrm{R}_{3}=0.04 \mathrm{H}_{\mathrm{d}}$. Moreover, the height of the dam, and the design head were respectively selected to be $\mathrm{H}_{0}=5 \mathrm{~m}$ and $\mathrm{H}_{\mathrm{d}}=1 \mathrm{~m}$.

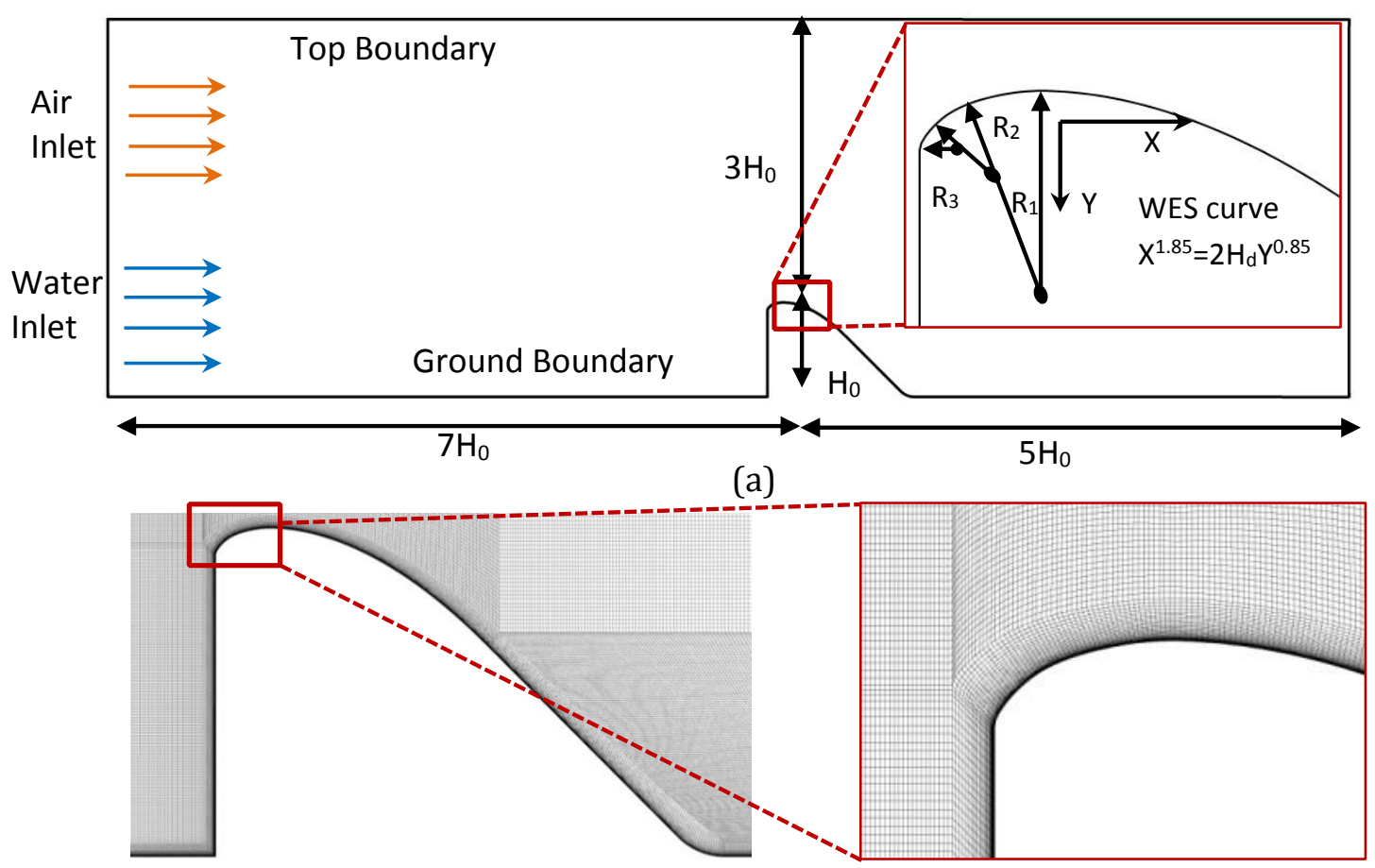

(b)

Figure 4 (a) CFD study domain and spillway characteristics (b) utilized grid with boundary layer cells

After choosing the case study, the 2D CFD model was validated using a series of measurements by the U.S. Army Corps of Engineers (1990). The assumption of creating a 2D model is fairly valid as the velocity variation is mainly in the flow direction. Several structured grids were generated for the CFD model to ensure the independency of the results from the grid 
size. It was observed that a mesh with $5.7 \times 10^{5}$ cells will not significantly improve the results. As illustrated in Fig. 4b, this grid was refined near the walls to ensure the wall y-plus is low enough for the enhanced wall treatment method, which guarantees representation of the boundary layer effect in the model (Mirzaei and Haghighat, 2013). Two transitional zones before and after the spillway are patched $5 \mathrm{H}_{0}$ and $7 \mathrm{H}_{0}$ to ensure that the water flow reaches the fully developed condition (Mirzaei and Carmeliet, 2013).

Table 1 Assigned boundary conditions of the hydraulic CFD model

\begin{tabular}{|c|c|c|}
\hline \multirow{2}{*}{$\begin{array}{c}\text { Inlet } \\
\text { boundary }\end{array}$} & Air inlet & Uniform velocity inlet, volume fraction $=0$ \\
\cline { 2 - 3 } & Water inlet & Uniform velocity inlet, volume fraction $=1$ \\
\hline \multicolumn{2}{|c|}{ Top boundary } & Symmetry \\
\hline Ground Boundary & Non-slip wall \\
\hline Outlet & Pressure outlet, open channel \\
\hline
\end{tabular}
and Haghighat, 2011). A separate velocity inlet boundary condition for the air and water was assigned to the upstream boundary. The volume flow rate for the water inlet was changed between $1 \mathrm{~m}^{3} / \mathrm{s}$ and $12 \mathrm{~m}^{3} / \mathrm{s}$. The material properties of both water and air were assumed to be constant at $20^{\circ} \mathrm{C}$. For the downstream boundary, the open channel model was utilized. The pressure-velocity coupling was achieved by SIMPLE algorithms. The PRESTO scheme was also used for the pressure interpolation. For steady-state calculations, the implicit scheme was used for the volume fraction equation. The momentum, volume fraction, and turbulent kinetic equations were discretised by the second-order upwind scheme while the turbulent intensity was assumed to be $10 \%$

\subsection{CFD validation}

As depicted in Fig. 5, the performance of the developed CFD model is evaluated by comparing the dimensionless static water head with the measured data by the U.S. Army Corps of Engineers, 1990. The result shows a close relation between the simulation and experimental results. Therefore, the verified grid and setup in this section will be used later as a basis for generation of other barriers. 


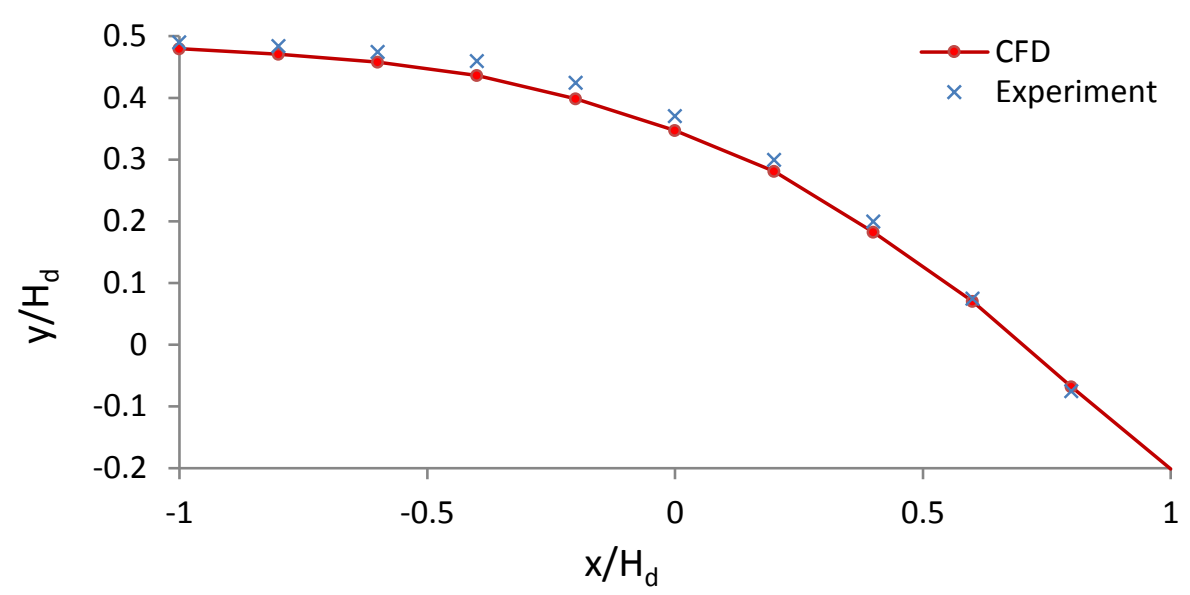

Figure 5 Static water head comparison between the measured data by the U.S. Army Corps of Engineers, 1990 and the developed CFD model

\subsection{Utilized parameters}

The fish jumping was determined by a number of physiological and behavioural parameters that vary with different species. Therefore, the framework should be initially calibrated by assigning the related parameters, e.g. starting location, buoyancy, drag and lift coefficients. The studied fish species is Salmon in this study as it is the most investigated species in back migration (Roscoe and Hinch, 2010). The weight and length of the selected Salmon were respectively considered to be $2 \mathrm{~kg}$ and $0.29 \mathrm{~m}$. The utilized drag and lift coefficients for the fish water kinematic model were obtained from a study by Takagi et al. (2013). These coefficients were defined to be a function of tail beating frequency, attack angle, and total body length. Moreover, the behavioural parameters were identified based on the previous studies in the literature and are presented in Table 2.

Table 2 Fish behavioural parameters adopted in swimming optimization model

\begin{tabular}{|l|l|}
\hline Fish behavioural Parameters & Description \\
\hline Response time & $\begin{array}{l}\text { The fish cannot respond instantly to the water velocity. Van Leeuwen }(1990) \\
\text { observed } 60 \mathrm{~ms} \text { time delay between the initial electrical stimulus and fully } \\
\text { mechanical response. }\end{array}$ \\
\hline Turbidity & $\begin{array}{l}\text { Nephelometric turbidity unit (NTU) is assumed to be 0.57 in clear water, and the } \\
\text { responding reactive distance is about } 0.9 \mathrm{~m} \text { (Sweka et al., 2001). }\end{array}$ \\
\hline Initial speed & $\begin{array}{l}\text { Reiser and Peocock (1985) identified three swimming speeds for various types of } \\
\text { fish, including cruising, sustained, and darting speeds. These speeds are reported } \\
0-0.61,0.61-1.95, \text { and } 1.95-4.11 \text { for Trout. The initial speed was assumed to be its } \\
\text { cruise swimming speed, 0.3m/s. }\end{array}$ \\
\hline Leaping speed $\left(V_{F}\right)$ & $\begin{array}{l}\text { According to Stuart (1962), the leap is mainly initiated at the point of the standing } \\
\text { wave. Thus, the vicinity of this point was inserted as a constraint to the swimming } \\
\text { optimization model. The leaping speed was also bounded between 0m/s and } \\
\text { 4m/s. }\end{array}$ \\
\hline Leaping angle $\left(\theta_{L}\right)$ & $\begin{array}{l}\text { It is reported through a series of experiments that salmons leave the pools with } \\
\text { their bodies straight and a combined mean take-off angle of 58 Degrees. This } \\
\text { angle is similar with the sea run fish observed by Lauritzen et al (2005). }\end{array}$ \\
\hline
\end{tabular}




\section{Results and discussion on the functionality of the leaping framework}

\subsection{Circulation importance}

To investigate the threshold of the hydraulic jump and formation of the back circulation in front of the case study spillway, the flow rate was changed between $0.5 \mathrm{~m}^{3} / \mathrm{s}$ and $6 \mathrm{~m}^{3} / \mathrm{s}$. This jump can be controlled based on the selection of a related dimensionless number, the Froude number (Fr). As illustrated in Fig. 6, a large back circulation was formed about a few meters after the crest where the hydraulic jump occurs. One of the main intentions of designing spillways is to avoid an intense back circulation right after the crest as this vortex, known as the drowning machine, caused thousands of casualties in the past years (Zamankhan, 2012). On the other hand, the current design of the spillway directs the flow with a high velocity slipping from the crest to the downstream, creating a high speed velocity region (Fig. 6). This region is an energy costly path for fish species to cross the barrier with either jumping or swimming. In both scenarios, fish consume a considerably high amount of energy to generate the required hydrodynamic forces to reach the upstream reservoir. Another feasible option to pass the barrier can be jumping over the back circulation of the hydraulic jump shown in Fig 6 . However, the far distance of this circulation from the upstream reservoir is normally beyond the jumping ability of fish species, implying that they need to swim toward the high speed region.

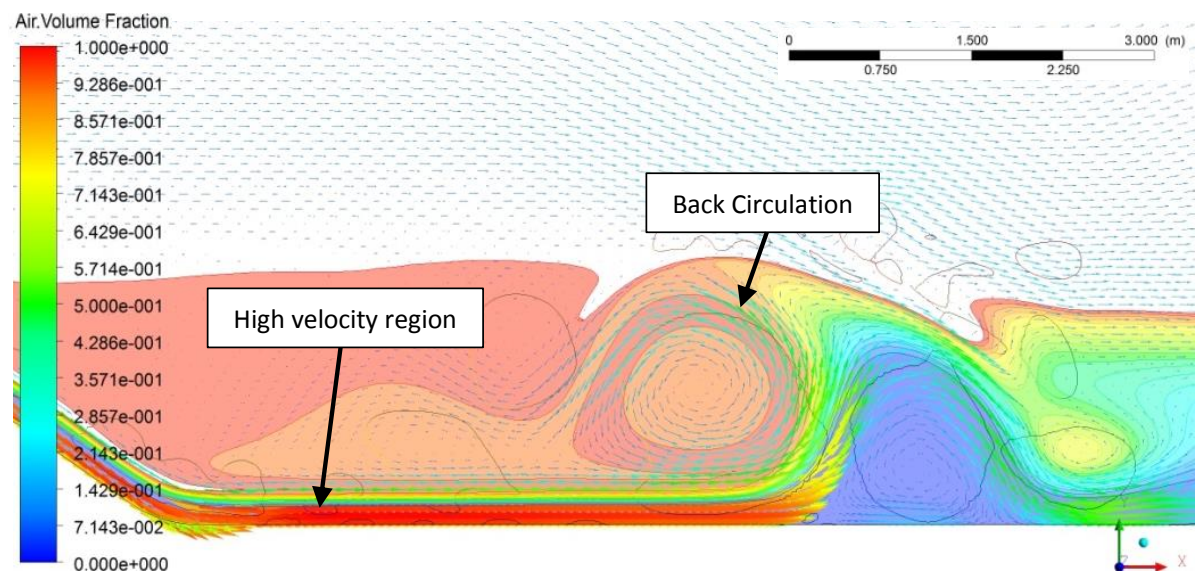

Figure 6 Back circulation and high velocity region in the studied spillway

As depicted in Fig. 7, it was observed that a small vortex in the vicinity of the spillway trail tends to appear in velocities lower than $5 \mathrm{~m}^{3} / \mathrm{s}$, leaving this region as an alternative to the high velocity region for the fish species to cross the spillway. Evidently, the size and strength of this 
vortex increases in the lower velocities. This means that the fish can benefit from this trail vortex where the upstream flow rate is low enough. On the contrary, the water level elevates in the higher flow rates, which may open a new option for fish species to benefit a better jump when they depart the water surface. As it was mentioned earlier, the aim of the framework is to consider the complex and dynamic regime of a water flow regime in the jumping of fish species.

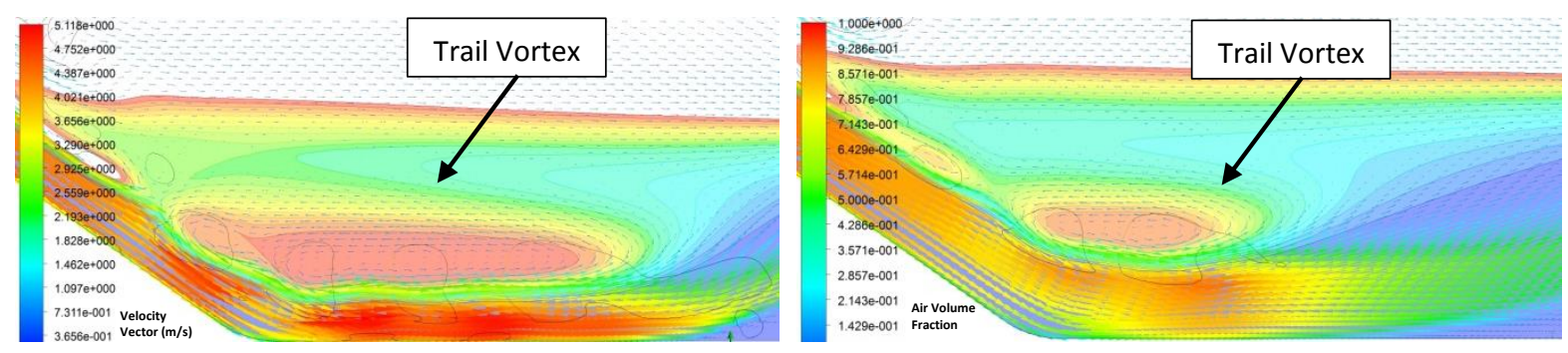

Figure 7 Impact of the air flow rate on the formation of the small vortex (left) $Q=2 \mathrm{~m}^{3} / \mathrm{s}$ (right)

$$
\mathrm{Q}=3 \mathrm{~m}^{3} / \mathrm{s}
$$

\subsection{Barrier shape}

The shape of a barrier significantly impacts the flow regime. In particular, the pool depth and waterfall heights could influence fish jumping when considering both characteristics simultaneously. As shown in Fig. 8a, a strong back circulation can be observed when the barrier has a different shape from a standard spillway as introduced before. Here, the fall height and pool depth are the key elements in characteristics (i.e. shape and strength) of this back circulation, impacting the jumping quality of the fish species.

To investigate the influence of the barrier shape on the jumping quality, a case study similar to an experiment by Lauritzen et al., (2010) is simulated with the CFD model. In this case, therefore, the fall height and the pool depth to fall height $(D / H)$ are respectively selected to be $0.36 \mathrm{~m}$ and $\mathrm{D} / \mathrm{H}=0.6$. Using the CFD model of Lauritzen et al., (2010) experiment shows that the preferred pool depth for jumping slightly increases with an increase in the fall height, but the preferred ratio of the pool depth to fall height decreases with an increase in the fall height. Adding a small ramp to the latter case scenario, it can be clearly seen in Fig. $8 \mathrm{~b}$ that the existence of a small ramp can change the shape and strength of the vortex as well as the elevation of the water flow. Therefore, it can be again concluded that the pool and barrier parameters noticeably 

be generated by fish species.
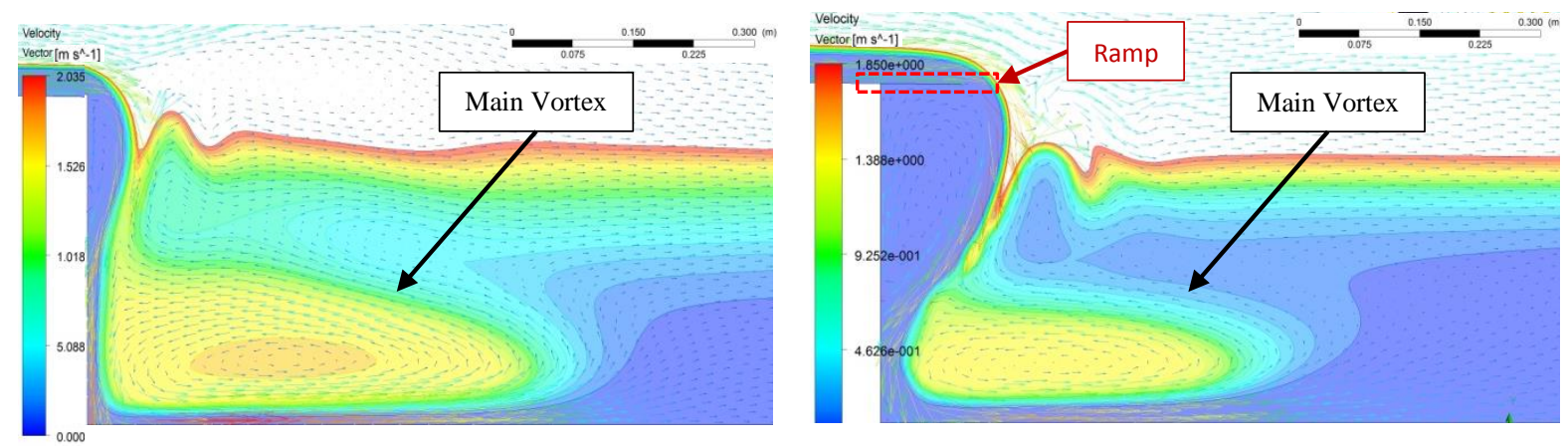

Figure 8 Impact of ramp on water flow regime (left) without ramp (right) with ramp

As it was explained by Fig. 1, the details of the water flow, which are unique for each

barrier, will be obtained at this stage and will be transferred to the fish water kinematic model to calculate the hydrodynamic forces at any location of the pool.

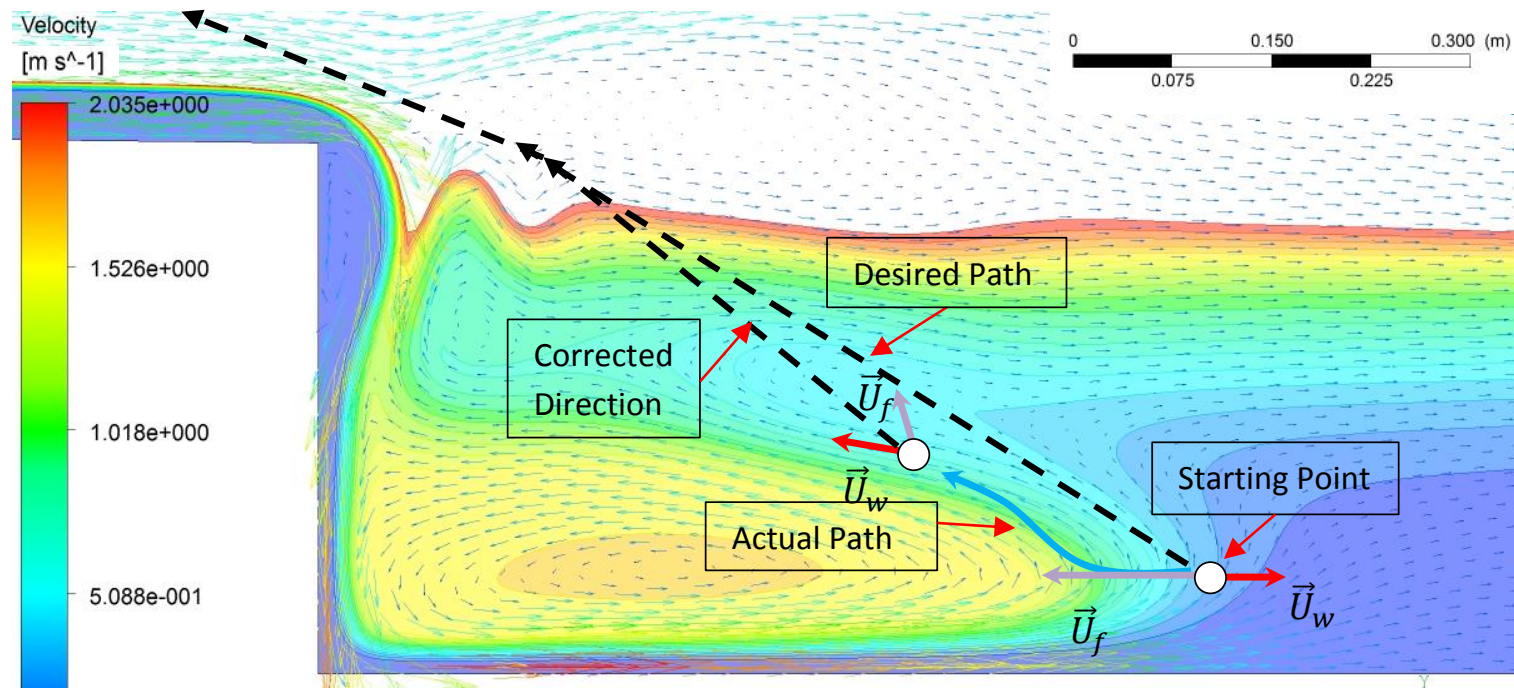

Figure 9 Desired and actual paths of the fish

5.3. Path optimization

The next step of the proposed framework is to simulate the jumping optimal solution from

the fish species point of view when the hydrodynamic forces are calculated from the previous steps. To minimize the swimming energy and ensure a successful jump, a fish can initially control its swimming velocity and angle. Before detecting the obstacle (low-head dam's wall), the fish continuously moves at cruise speed in the horizontal direction. When the obstacle is detected in accordance with the turbidity number (Table 2), it decides to initiate the jumping process (Fig. 
3). It is noteworthy to mention that the selection of initial point of jumping significantly impact the optimization result. In this study, the ending of the horizontal cruse is used as the starting point for the optimization calculation (Fig. 9). Therefore, this point is located where the fish deviates from its previous straight line. Fish determine their desired path by visual stimulus, meaning that it constantly revises its path and velocity toward the water surface.

As it is shown in Fig. 9, the fish deviates from its desired path due to the sudden decrease in drag force due to the time lag, while the propulsion force is still fixed. This indicates that the total force applied on the fish cannot lead it to the desired path, but towards the direction of water velocity, sketching an actual path for the fish. This continuous adjustment process is calculated in the swimming optimization model. The locomotion is moreover assumed to be accomplished in several time steps in respect to the response time of a fish species. This means that a fish can modify the current path after each response time. Thus, in each node, the model simulates the largest exerted thrust force by the fish to accelerate itself for a successful jump over the spillway as depicted in Fig. 3. Then the algorithm investigates the possible scenarios of the current node. This includes whether the fish (1) hits the wall (Fig. 10a), implying an unsuccessful attempt, (2) swims backward when the water velocity is too large to be dominated, (3) departs the water surface where the swimming optimization model will transfer the direction, speed, and location of the fish to the Air Trajectory Model in order to evaluate the quality of the jump (Fig. 10b and c), or (4) relocate to a new point toward the barrier, meaning the generation of three new nodes (Fig. 3). Eventually, all the nodes will be generated toward the top surface boundary where the calculation will stop. The selection of three new nodes is due to reducing the computational time of the optimization algorithm where the angle between thrust force and fish swimming velocity is assumed to be less than 30 degrees (Pearson et al. 2005).

The fish control strategy is affected by a variety of factors, including age, sex, temperature, burst speed, starting location of the jump and turbulence. Hence, it is assumed that the fish is able to relocate between nodes by reduction and elevation of the thrust force. Utilizing this concept, the optimization model can find the critical thrust, the minimum thrust required for the fish to 

critical thrust is then assumed as a failed jump attempt.

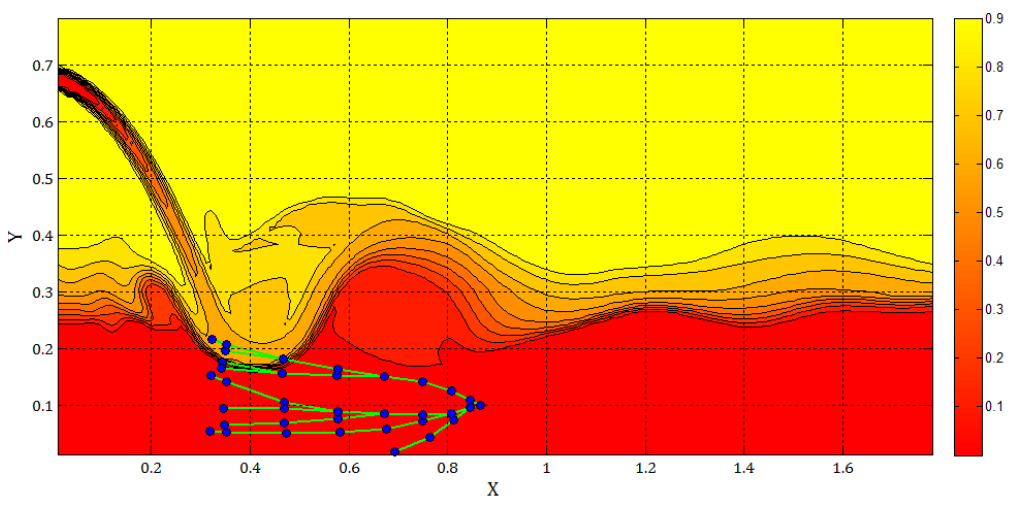

(a)

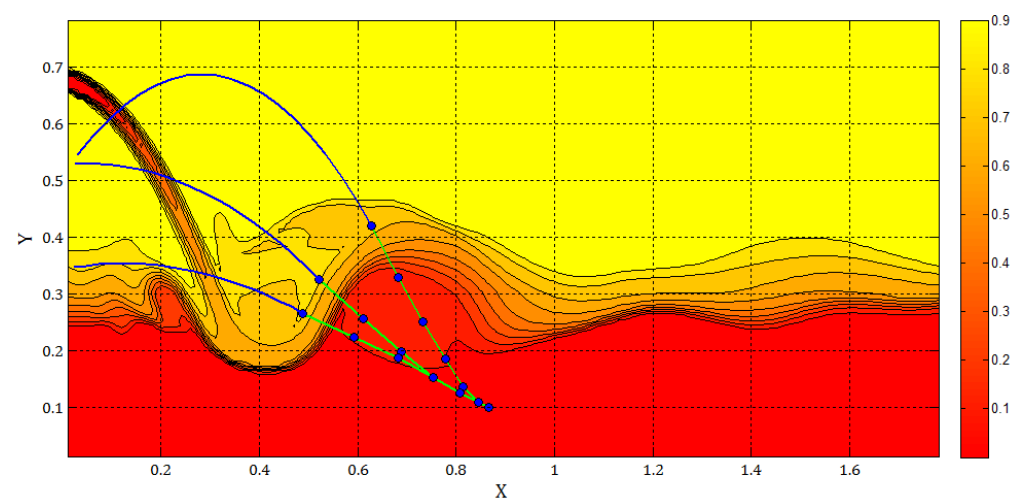

(b)

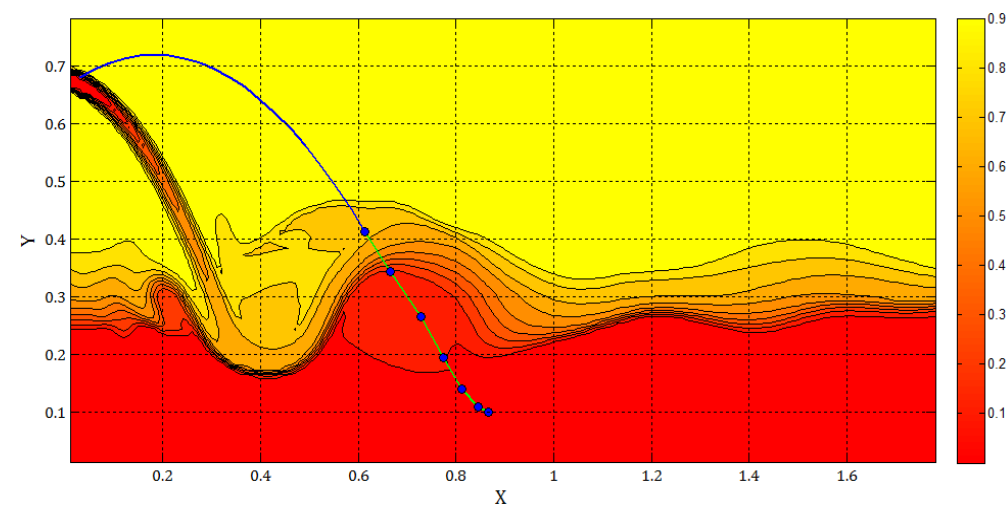

(C)

Figure 10 Example of the possible scenarios for a specific barrier and water flow (a) incomplete jumps

(b) jump failures (c) a successful jump

These scenarios are illustrated in Fig. 10 associated with the defined case study in the previous section $(\mathrm{H}=0.36 \mathrm{~m})$. Some of the paths in which the fish is tapped under the water fall due to its miscalculation on the direction and thrust generation are shown in Fig. 10a. Similarly, Fig. 10b demonstrates the paths in which the fish reaches the water surface, but without a suitable burst speed or direction to reach the upstream reservoir. Eventually, one of the feasible successful 

suitable directions on each node toward the water surface. species. For example, in the above case study, the probability of the leaping should be initially calculated according to the various leaping scenarios as explained in the previous sections - this value is below acceptable range with various assumed inputs to the framework related to the fish species and its behaviour. The obtained probability thus empower the decision makers to apply a variety of strategies to change or retrofit the barrier, e.g. alteration of the outfall drop, plunge pool depth, water flow rate, etc.

\section{Conclusion}

A fish leaping framework, including hydraulic CFD, fish water kinematic, swimming optimization, and fish air trajectory models, is proposed in this study. The proposed framework is initially validated with physical characteristics of a barrier and calibrated with behavioural and physiological parameters of a fish species. The capability of the developed model in calculating the leaping success rate of the fish is then shown with a case study. The results clearly show that the framework is capable of the calculation of the favourability of a specific barrier.

As a future study, the developed framework can be improved by including the learning process of the fish species. Moreover, the framework can be utilized to calculate the total energy required for a complete upstream spawning migration of a fish species. Furthermore, more research can be conducted for better understanding of the behavioural parameters that contribute to the leaping process of a fish species.

\section{Acknowledgment}

The author would like to extent his gratitude to Mr. Hanxiao Cui, Mr. Mou Chen and Mr. Michael Trojan for their help through this study.

\section{References}

\footnotetext{
Carpenter, R. E., Summers, C. H. (2009) Learning strategies during fear conditioning. Neurobiology of Learning and Memory. 91(4):415-23.
} 
Enders, E. C., Boisclair, D., Roy, A. G. (2003). The effect of turbulence on the cost of swimming for juvenile Atlantic salmon (Salmo salar). Canadian Journal of Fisheries and Aquatic Sciences. 60, 1149-1160.

Ficke, A. D., Myrick, C. A., Jud, N. (2011) The Swimming and Jumping Ability of Three Small Great Plains Fishes: Implications for Fishway Design. Transactions of the American Fisheries Society, 140:6, 1521-1531.

Gehrke, P. C., Gilligan, D. M., Barwick, M. (2002) Changes in fish communities of the Shoalhaven River 20 years after construction of Tallowa Dam, Australia. River Research and Applications 18:265-286.

Holthe, E., Lund, E., Finstad, B., Thorstad, E. B., McKinley, R. S. (2005) A fish selective obstacle to prevent dispersion of an unwanted fish species, based on leaping capabilities. Fisheries Management and Ecology. 12 143-7.

Kondratieff, M. C., Myrick, C. A. (2006) How High Can Brook Trout Jump? A Laboratory Evaluation of Brook Trout Jumping Performance. Transactions of the American Fisheries Society. 135:361-370.

Lauritzen, D. V., Hertel, F., Gordon, M. S. (2005) A kinematic examination of wild sockeye salmon jumping up natural waterfalls. Journal of Fish Biology. 67 1010-20.

Lauritzen, D. V., Hertel, F. S., Jordan, L. K., Gordon, M. S. (2010) Salmon jumping: behaviour, kinematics and optimal conditions, with possible implications for fish passageway design. Bioinspiration \& Biomimetics. 5(3):035006.

Liao, J. C. (2007) A review of fish swimming mechanics and behaviour in altered flows. Philosophical Transactions of the Royal Society B. 362, 1973-1993.

Liao, J. C., Beal, D. N., Lauder, G. V., Triantafyllow, M. S. (2003) Fish Exploiting Vortices Decrease Muscle Activity. Science. 302, 1566.

McDowall, R.M. (1990) New Zealand Freshwater Fishes: A Natural History and Guide. Heinemann Reed, Auckland.

Marmulla, G. (ed.). (2001) Dams, fish and fisheries. Opportunities, challenges and conflict resolution. FAO Fisheries Technical Paper. No. 419. Rome, FA0. 166p.

McLaughlin, R. L., Smyth, E. R. B., Castro-Santos, T., Jones, M. L., Koops, M. A., Pratt, T. C., Ve'lezEspino, L.A. (2013) Unintended consequences and trade-offs of fish passage. Fish and Fisheries, 14, 580604.

Mirzaei, P.A., Haghighat, F. (2011) Pollution removal effectiveness of the pedestrian ventilation system. Journal of Wind Engineering \& Industrial Aerodynamics, Journal of Wind Engineering and Industrial Aerodynamics, 99: 46-58.

Mirzaei, P.A., Carmeliet, J., (2013) Dynamical Computational Fluid Dynamics Modeling of the Stochastic Wind for Application of Urban Studies. Building and Environment, 70:161-170.

Mirzaei, P.A., Rad, M. (2013) Toward design and fabrication of wind-driven vehicles: procedure to optimize the threshold of driving forces, Applied Mathematical Modelling, 37: 50-61.

Myrick, C., Kondratieff, M. (2004) An Evaluation of a Potential Barrier to the Upstream Movement of Brook Trout in Rocky Mountain National Park, Colorado.

Noonan, M. J., Grant, J. W. A., Christopher D. J. (2012) A quantitative assessment of fish passage efficiency. Fish and Fisheries, 13, 450-464.

Odling-Smee, L., Braithwaite, V.A. (2003) The role of learning in fish orientation. Fish and Fisheries, 4, 235-246.

Powers, P. D., Orsborn, J. F. (1985) Analysis of barriers to upstream fish migration. Albrock Hydraulics Laboratory, Contract DE-A179-82BP36523, Project 82-14, Pullman, Washington.

Pavlov, D. S., Lupandin, A. I., Skorobogatov, M. A. (2000) The effects of flow turbulence on the behavior and distribution of fish. Journal of Ichthyol. 40, S232-S261.

Pavlov, D. S., Skorobogatov, M. A., Shtaf, L. G. (1982) The critical current velocity of fish and the degree of flow turbulence. Rep. USSR Academy of Science. 267, 1019-1021.

Pearson, W. H., Mueller, R. P., Sargeant, S. L., May, C. W. (2005) Evaluation of Juvenile Salmon Leaping Ability and Behavior at an Experimental Culvert Test Bed. Prepared for: Washington State Department of Transportation. WSDOT Agreement No. GCA2677. 
Reiser, D. W., Peacock, R. (1985) A technique for assessing upstream fish passage problems at small-scale hydropower developments. Pages 423-432 in F. Olson, R. White, and R. Hamre, editors. Proceedings of the symposium on small hydropower and fisheries. American Fisheries Society, Bethesda, Maryland.

Reiser, D. W., Huang, C.M., Beck, S., Gagner, M., Jeanes, E. (2006) Defining Flow Windows for Upstream Passage of Adult Anadromous Salmonids at Cascades and Falls. Transactions of the American Fisheries Society 135: 668-679.

Roscoe, D. W., Hinch, S. G., (2010) Effectiveness monitoring of fish passage facilities: historical trends, geographic patterns and future directions. Fish and Fisheries, 11, 12-33.

Schlosser, I. J., Angermeier, P. L. (1995) Spatial variation in demographic processes of lotic fishes: conceptual models, empirical evidence, and implications for conservation. American Fisheries Society. 392-401.

Sweka, J. A., Hartman, K. J. (2001) Influence of Turbidity on Brook Trout Reactive Distance and Foraging Success. Transactions of the American Fisheries Society 130: 138-146.

Takagi, T., Tamura, Y., Weihs, D. (2013) Hydrodynamics and energy-saving swimming techniques of Pacific Bluefin tuna. Journal of Theoretical Biology 336, 158-172.

U.S. Army Corps of Engineers, (1990) Hydraulic Design of Spillways.

Wilcox, B. A. (1980) Insular ecology and conservation. Pages 95-118 in M. E. Soulé and B. A. Wilcox, eds. Conservation biology: an evolutionary-ecological perspective. Sinauer, Sunderland, MA.

Wilcox, B. A., Murphy, D. D. (1985) Conservation strategy: the effects of fragmentation on extinction. American Naturalist 125:879-887.

Webb, P. W. (1998) Entrainment by river chub Nocomis micropogon and smallmouth bass Micropterus dolomieu on cylinders. Journal of Experimental Biology. 201, 2403-2412. 48:215-229.

Weihs, D. (1973) Energetic Advantages of Burst Swimming of Fish. Journal of Theoretical Biology

Van Leeuwen, J. L., Lankheet, M. J. M., Akster, H. A., Osse, J. W. M. (1990) Function of red axial muscles of carp (Cyprinus carpio): recruitment and normalized power output during swimming in different modes. Journal of Zoology 220, 123-145.

Zamankhan, P. (2012) Rollers in low-head dams - Challenges and solutions. Communications in Nonlinear Science and Numerical Simulation. Volume 17, Issue 12, Pages 5273-5285. 